\title{
La cultura profesional del docente en un contexto de cambios: retos y perspectivas
}

\section{The professional culture of the educational one in a context of changes: challenges and perspectives}

\author{
Dr. C Lázara Bastida Lugones ${ }^{1}$ \\ lbastidalugones@gmail.com \\ https://orcid.org/0000-0002-0749-570X \\ Dr. C Alina Rodríguez Morales ${ }^{2}$ \\ alina.rodriguezm@ug.edu.ec \\ https//orcid.org/0000-0003-12009-8802 \\ Ms C Betsy Alvarez Vega ${ }^{3}$ \\ balvarez@ucf.edu.cu \\ https://orcid.org/0000-0002-6824-2271
}

Recibido: 1/4/2020, Aceptado: 1/6/2020

\begin{abstract}
RESUMEN
El artículo revisa trabajos conceptuales sobre el actual contexto del proceso educativo. Toma como referente la apertura hacia nuevos horizontes, así como los entornos virtuales de aprendizaje en los que la escuela ha perdido el monopolio, se abren nuevas perspectivas y exigen el desarrollo de planes formativos y nuevos planteamientos de la función y formación del profesorado. El propósito de la revisión de literatura es el de comparar, contrastar y organizar elementos dentro de la cultura científico-investigativa que los docentes enfrentan día a día. De esa manera, se concluye en material provechoso para el profesional que enfrenta cambios profundos en los últimos años en la llamada sociedad de la información, así como las modificaciones continuas a las que se ve sometido el sistema educativo, las exigencias de la sociedad, las transformaciones de las mentalidades y las nuevas demandas educativas, el desarrollo de contextos educativos diferentes a los que tradicionalmente se han venido utilizando. Las tecnologías de la información y las comunicaciones hacen evidente la necesidad de búsqueda de modelos formativos actuales para el profesorado que ofrezcan respuestas óptimas al reto que los tiempos modernos les plantea.
\end{abstract}

Palabras clave: proceso educativo, formación del profesorado, contextos educativos, tecnologías de la información y las comunicaciones

\footnotetext{
${ }^{1}$ Instituto Central de Ciencias Pedagógicas, La Habana, Cuba.

2 Universidad de Guayaquil, Guayaquil, Ecuador.

${ }^{3}$ Universidad Carlos Rafael Rodríguez, Cienfuegos, Cuba.
} 


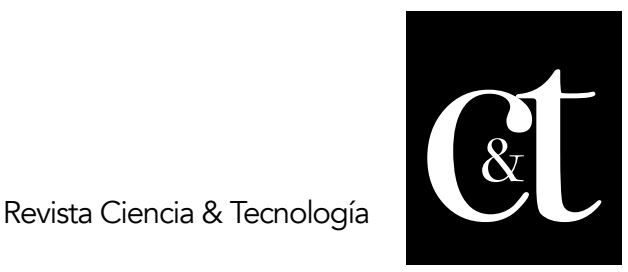

No. 27, 31 de julio de 2020

ISSN impreso: 1390 - 6321

ISSN online: 2661 - 6734

\begin{abstract}
This article reviews conceptual works on the current context of the educational process. It takes as a reference the opening towards new horizons, as well as the virtual learning environments in which the school has lost its monopoly, open new perspectives and demand the development of training plans and new approaches to the role and training of teachers. The purpose of this work review is to compare, contrast and organize elements within the scientific research culture that teachers face every day. In this way, it is concluded in useful material for the professional who faces profound changes in recent years in the so-called information society, as well as the continuous modifications to which the educational system is subjected, the demands of society, the transformations of mentalities and new educational demands, the development of educational contexts different from those that have traditionally been used. Information and communication technologies make evident the need to search for current training models for teachers that offer optimal responses to the challenge that modern times pose to them.
\end{abstract}

Keywords: educative process, teacher formation, educative context, information and communication techniques

\title{
Introducción
}

En el actual contexto educativo la formación del profesor va unida al desarrollo profesional del docente. Esto no constituye una cuestión nueva, se trata de concebir la formación como un aprendizaje constante, de modo que la formación se acerque al desarrollo de actividades profesionales y a la práctica profesional y desde ella. Desde esta perspectiva se considera, por tanto, la práctica de la enseñanza como una profesión dinámica, en continuo desarrollo, por ello, la formación y el reciclaje son aspectos muy importantes que todos los profesores deben asumir como propios.

El desarrollo de una cultura profesional tiene que ver en el profesorado con los conocimientos existentes y la concepción o función docente, con las orientaciones conceptuales de su función, con la formación inicial, con el status y las condiciones de trabajo, con la formación permanente, con la evaluación a la que se somete su trabajo, y con otros procesos paralelos que afectan igualmente al desarrollo de la cultura profesional del profesorado y, consecuentemente, al proceso de innovación y cambio educativo (UNESCO, 2005; UNESCO-PRELAC, 2007).

A través de la revisión de literatura científica se ve el actual contexto de cambios en el ámbito social que tienen una repercusión en el ámbito escolar. Asimismo, la formación y la cultura profesional del docente, ha cambiado. Ya no basta con saber, sino que también es necesario un saber vinculado con los profundos cambios económicos y sociales en marcha, con las tecnologías de la información y las comunicaciones, con la nueva organización industrial e institucional, en un mundo crecientemente complejo e interdependiente, que requiere personas con viva inquietud creativa e innovadora, con espíritu crítico, reflexivo y participativo (López Rodríguez del Rey \& Bastida, 2006).

El papel del profesor en los espacios educativos virtuales y en los espacios educativos tradicionales va más allá de ser transmisores, los profesores deben asumir otros roles, y esto requiere diferentes estrategias de enseñanza. Weil, Joyce y Kluwin (1978) establecen diversos modos de organizar las estrategias y estilos de 


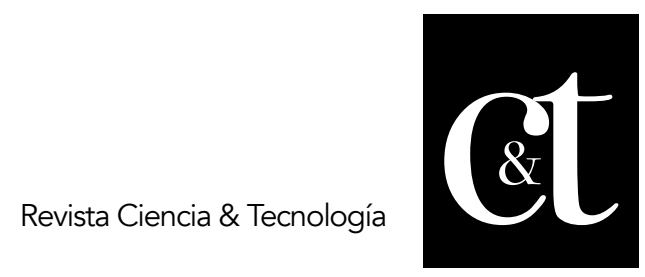

No. 27, 31 de julio de 2020

ISSN impreso: 1390 - 6321

ISSN online: 2661 - 6734

enseñanza, indicando diferentes modelos: modelo de interacción social, modelos de procesamiento de la información, modelos personales y modelos de modificación de conducta (Weil, Joyce y Kluwin, 1978). Sin embargo, ninguno de estos modelos resulta concluyente, ya que la dificultad y complejidad del proceso de enseñanzaaprendizaje y las diferentes relaciones entre profesores y estudiantes hacen muy difícil establecer un modelo o estilo infalible. Más bien se debe intentar re -asumir todas estas teorías y establecer un modelo propio que sea capaz de aglutinar la idiosincrasia del proceso de enseñanza-aprendizaje en las aulas.

\section{Desarrollo}

Desde esta perspectiva se asume con racionalidad pedagógica y se advierte que la educación es una acción y es un efecto. La palabra educación es muy genérica, ya que encierra todo hecho o fenómeno que provoca cambios en la modificación de la conducta del individuo, es decir, es un proceso intencionado de formación de las nuevas generaciones. En este sentido, la educación puede plantearse como una actividad formativa, como una influencia o manipulación, como una realidad íntima e individual, como un proceso socio-cultural.

Interesa en este particular destacar que la educación es una acción pensada, meditada, reflexionada, intencional, organizada y sistematizada, con un claro objetivo, con unas metas centrales y colaterales. De este modo interesa referirse a los términos de acción pedagógica y de racionalidad pedagógica por la importancia que reviste para las autoras del presente artículo.

La racionalidad pedagógica, parte del presupuesto de entender la educación, en este caso en concreto, como una acción o proceso intencionado, con una clara organización y estructura. En tanto la acción pedagógica es un hacer, no un pensar, puesto que se trata de intervenir específicamente en entornos sociales y educativos concretos. La racionalidad pedagógica se concreta a través de un fenómeno reflexivo, que averigua, indaga y formaliza la acción educativa como un fenómeno de la comunicación humana. Es, por lo tanto, la acción pedagógica un proceso, generalmente, intencional, para el que es preciso un cierto nivel de inteligencia y esfuerzo, que implica aprender y que debe estar orientado al mundo de los valores, las creencias y las ideologías.

En virtud de este mundo de valores y creencias, la acción educativa debe replantearse en una estructura axiológica o ideológica que permita al estudiante alcanzar un desarrollo moral que le ofrezca la seguridad de poder posicionarse críticamente ante el mundo, y utilizar su capacidad crítica para tomar parte activa del mundo y en particular de la educación (Romero-Martín, Castejón-Oliva, López-Pástor y FraileAranda, 2017).

Por ello, es preciso recordar que la acción educativa debe incidir en la fenomenología global del hombre, es decir, que debe ser integral. La intervención educativa debe estar orientada hacia el estudiante, auténtico, protagonista del acto didáctico. La orientación hacia el estudiante hace que el profesor se replantee sus estilos educativos, que innove, que enseñe al estudiante a vivir, en definitiva, que readapte y tome conciencia de su cultura docente y su profesionalidad.

Nótese como estos aspectos abordados con anterioridad presuponen ampliar la perspectiva de la cultura del docente y la profesionalidad del profesor, por ello, se asumen las concepciones de Jacques Delors (1996) quién apunta que el trabajo del 


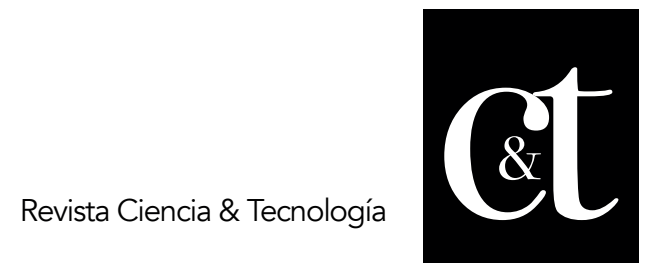

No. 27, 31 de julio de 2020

ISSN impreso: 1390 - 6321

ISSN online: 2661 - 6734

docente no consiste tan sólo en transmitir información ni siquiera conocimientos, sino en presentarlos en forma de problemática, situándolos en un contexto y poniendo los problemas en perspectiva, de manera que el estudiante pueda establecer el nexo entre su solución y otras interrogantes de mayor alcance". El profesor se convierte en mediador del acto didáctico. Y mediador del acto didáctico significa mediar entre el discente y los contenidos de la asignatura. Así, el profesor media entre el contenido y el estudiante, de modo que las estrategias y estilos de enseñanza repercuten en el rendimiento académico del estudiante. Por lo tanto, en el actual contexto educativo, el papel del profesor no debe reducirse a la mera transmisión de conocimientos, sino que se orienta a desarrollar la labor educativa y por consiguiente la formación integral de los estudiantes. Se coincide con Salvador Mata (1994) quien plantear la docencia como compleja y difícil; sin embargo, resulta gratificante en la mayoría de las ocasiones.

El rol docente en las condiciones actuales de cambio donde se requiere una transformación de sus modos de actuación implica en primer lugar considerarse a uno mismo como educador, de modo que garantice la labor educativa de sus estudiantes. Ser un docente no implica dedicarse a dictar apuntes y consumir sus horas de clases, sino en entregarse en cada una de ellas, en descubrir en sus estudiantes un universo de conocimientos sin explorar (Pino, 2005).

Ello exige, no solo ocupación profesional, sino también una vocación de servicio hacia el alumnado. El verdadero docente se esfuerza cada día en su labor educativa para que sus estudiantes utilicen sus recursos y hagan el mejor uso posible de sus posibilidades y potencialidades. Actualmente, desde una perspectiva pedagógica abierta y actual, el profesor tiene más responsabilidad en tareas de motivación, orientación y educación, que van más allá de las meramente instructivas.

El rol docente, la verdadera esencia y responsabilidad del profesor no pasa por inculcar respuestas e imponer contenidos, fórmulas matemáticas, datos estadísticos, hechos históricos, conceptos, entre otros aspectos. La cultura del docente y su rol en el actual contexto de cambios va más allá, es decir dirigido a orientar a los estudiantes hacia el descubrimiento y la autonomía, proporcionándoles las herramientas para que adquieran una conducta y posición crítica ante el mundo y ante ellos mismos, que les haga interrogarse permanentemente. Es formar individuos críticos, libres, democráticos, innovadores, trabajadores, responsables, participativos, humanos, sociales.

En tal sentido para elevar la cultura profesional del docente se requieren cambios en sus métodos y estilos de enseñanza, de manera que los adapten a los nuevos tiempos y a las demandas actuales de los estudiantes, que buscan en el profesor el vínculo, el enlace entre lo desconocido y sus propias capacidades. Por este motivo, la personalidad y los estilos de enseñanza del profesor deben adaptarse (en la medida de lo posible) a todos y cada uno de los estudiantes, es decir, el profesor debe convertirse en un guía, orientador y modelo del proceso de enseñanza-aprendizaje, en lo que se ha denominado mediador del acto didáctico.

Sobre este particular se coincide con los criterios abordados por Fernández y Sarramona (1977) sobre las condiciones y cualidades fundamentales que deben caracterizar a un docente en su desempeño profesional entre las que se destacan las siguientes: 


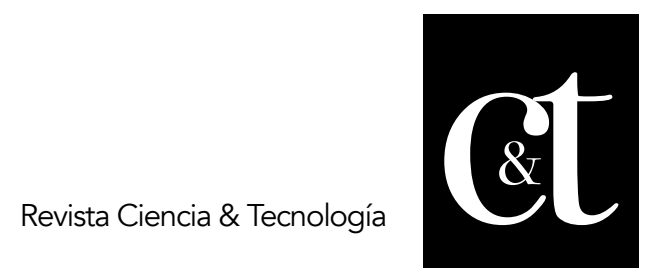

No. 27, 31 de julio de 2020

ISSN impreso: 1390 - 6321

ISSN online: 2661 - 6734

1. Capacidad de conocimiento y adaptación a los estudiantes, tanto individual como colectivamente, para lo que es necesario contar con una personalidad madura y equilibrada.

2. Conocimientos didácticos y pedagógicos necesarios para adaptar los contenidos a las necesidades y características de los estudiantes.

Sin lugar a dudas el rol del docente debe configurar al profesor como el profesional de la enseñanza que demuestra poseer flexibilidad, adaptación, sensibilidad, con capacidad para dar respuestas a los problemas que se encuentra en el aula (profesionales y emocionales), empático, objetivo, capaz de observar lo que está ocurriendo en el contexto instruccional, auténtico y sincero, no dominante, ni directivo, ni autoritario, capaz de no intervenir cuando no sea necesario ni se lo pidan, que intervenga para que el estudiante participe y aprenda, con una actitud positiva, abierto al cambio y a la innovación, capaz de comunicarse y transmitir de forma eficaz y positiva, y por supuesto, tan implicado en su trabajo que posea una fuerte y profunda motivación intrínseca.

Que transmita, en definitiva, la alegría por aprender, y que sea capaz de conseguir en sus estudiantes una motivación de aprendizaje, de modo que ellos mismos se sientan seguros y cómodos en sus clases (Castellanos, 2006; Del Pino, 2008).

Ello requiere trabajar en función de que los estudiantes desarrollen competencias para elegir, tomar decisiones, elaborar planes y proyectos de vida, para que dominen los instrumentos que les permitan autorregularse en los diferentes ámbitos de su vida. Es necesario para favorecer el cambio en virtud de la calidad de la educación que el profesor eleve su cultura profesional y, además:

- Cree una atmósfera de confianza, seguridad y empatía en el aula, para que su trabajo repercuta en todas las esferas de la personalidad de los estudiantes: intelectual, emocional, motivacional, volitiva, que regulen favorablemente su desempeño.

- Organice situaciones de aprendizaje basadas en problemas reales, significativos, que favorezcan el desarrollo de motivaciones intrínsecas, con niveles de desafío adecuados, acordes a la zona de desarrollo potencial de sus estudiantes.

- Apoye a los estudiantes para que acepten los retos del aprendizaje y aprendan a identificar y resolver problemas, favoreciendo que estos seleccionen e implementen sus propios caminos de solución y brindando las ayudas oportunas y necesarias, individualizadas a la situación de cada sujeto. En ese sentido es importante que el profesor sirva de modelo en la búsqueda y aplicación de estrategias efectivas para la resolución de problemas.

- Propicie la participación de todos los miembros del grupo según sus potencialidades, animando a los más pasivos y cuidando que ninguno monopolice la atención; creando una estructura cooperativa de trabajo en el aula en la cual se logren compromisos individuales y grupales en función del logro de metas comunes; facilitando la comunicación de ideas mediante la resolución de problemas en grupo; actuando como moderador y facilitador, pero también como tutor, experto y supervisor.

- Emplee el error con fines educativos y estimule la atribución consciente de los éxitos y fracasos escolares a causas controlables, modificables; creando espacios de autoconocimiento, donde los estudiantes se entrenen en la autorreflexión y aprendan a observarse, interrogarse, analizar alternativas y consecuencias, tomar decisiones, plantearse objetivos, aspiraciones, y analizar sus posibilidades reales 


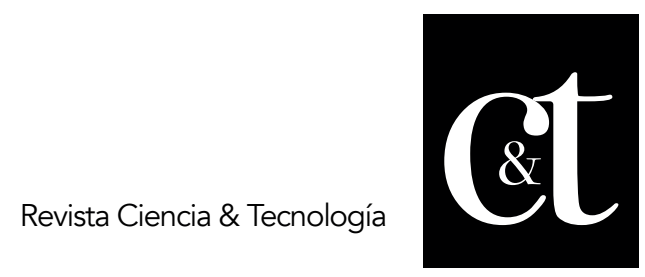

No. 27, 31 de julio de 2020

ISSN impreso: 1390 - 6321

ISSN online: 2661 - 6734

de alcanzarlas. Con todo ello favorece la reflexión y autorregulación metacognitiva.

- Diagnostique fortalezas, dificultades y potencialidades en el aprendizaje de sus estudiantes y, sobre esa base, conciba estrategias de enseñanza, diferenciadas y desarrolladoras, para cada miembro en particular y en general para el grupo, apoyándose en la combinación complementaria del aprendizaje cooperativo y el aprendizaje independiente.

- Pero la labor de orientación del docente no va dirigida solo a los estudiantes sino a todos los agentes educativos, por tanto, también al grupo; el colectivo de docentes, directivos y demás trabajadores del centro; a la familia y otras instancias de la comunidad, que como agentes de cambio deben trabajar de manera coordinada, como un sistema de influencias educativas.

- Para alcanzar tales propósitos se requiere la participación consciente de cada uno de ellos, en particular de los estudiantes y el grupo, que se conciban como agente de cambio y consecuentemente se impliquen en esos procesos y se comprometan con dicho propósito. Es labor del profesor -como docente o directivo- coordinar esas influencias educativas y trabajar en función de que se integren, de que funcionen como un sistema.

- La orientación no es una tarea puntual, de determinados momentos o etapas sino un proceso permanente y exige del profesor un desempeño en el que se aproveche la diversidad, combinándose la atención individual y grupal.

\section{Conclusiones}

La cultura del docente para enfrentar estos retos planteados a la educación aún es deficiente $y$, en consecuencia, la investigación educativa no juega su verdadero papel en la transformación de su práctica y en su crecimiento profesional.

Es evidente que desde esta concepción se privilegia la cultura científico-investigativa del docente con un carácter globalizador: el docente no solo debe saber de sus ciencias, sino también de aquellas concomitantes que contribuyen a la formación del estudiante, de manera que desarrolle una práctica educativa reflexiva y problémica, que se transforme sistemáticamente y sea generadora del saber pedagógico bajo la conducción del método científico.

La cultura científico-investigativa aún no ha penetrado orgánicamente en el diseño curricular de su formación continua. Por tanto, aún no es inherente a su práctica cotidiana. Por ello, estas propuestas deben ser incorporadas en la formación de los profesionales de la educación en aras de armonizar el proceso educativo en el actual contexto de cambios que se generan en el ámbito socio-cultural y, de esta forma, elevar su cultura profesional.

\section{Referencias bibliográficas}

Castellanos, D. (2006). Aprender y enseñar en la escuela. Editorial Pueblo y Educación. Ciudad de La Habana.

Delors, J. (1996). La Educación encierra un tesoro. Madrid: Santillana.

Del Pino, J. L. C. (2008): La orientación profesional pedagógica: el reto de su perfeccionamiento. Editorial Pueblo y Educación. Ciudad de La Habana.

Fernández, A. y Sarramona, J. (1977). La educación. Constantes y problemática actual. Barcelona: CEAC. 


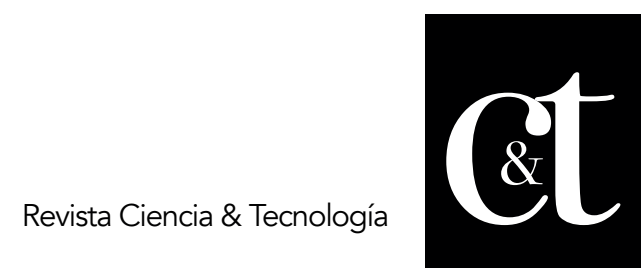

No. 27, 31 de julio de 2020

ISSN impreso: 1390 - 6321

ISSN online: 2661 - 6734

López Rodríguez del Rey, M. \& Bastida, L. (2006). La integración: claves para una práctica en las condiciones de universalización en la formación docente en Cienfuegos. Ponencia presentada en el evento provincial Universidad, 2006. Cienfuegos: MINED.

Pino L. M. (2005). La cultura científica: una necesidad del proceso de enseñanza aprendizaje en el siglo XXI. Ponencia. 5to Congreso Provincial de Educación Superior. Ciudad de la Habana.

Romero-Martín, R., Castejón-Oliva, F. J., López-Pastor, V. M., \& Fraile-Aranda, A. (2017). Evaluación formativa, competencias comunicativas y TIC en la formación del profesorado. Comunicar: Revista Científica de Comunicación y Educación, 25(52), 73-82.

Salvador Mata, F. (1994). El profesor como mediador en el acto didáctico. En O. Sáenz Barrio (Dir.): Didáctica General. Un enfoque curricular. Alcoy: Marfil, pp. 71-72.

UNESCO (2005). Decenio de las Naciones Unidas de la Educación para el desarrollo sostenible (2005-2014).

UNESCO-PRELAC (2007). Educación de calidad para todos: asunto de derechos humanos. Buenos Aires.

Weil, M.; Joyce, B. y Kluwin, B. (1978). Personal models of teaching. New Jersey: Prentice-Hall. 